\title{
Les urgences : « trop cher... vous avez dit trop cher ?... »
}

\author{
Emergency: "too expensive... you said too expensive?..."
}

\author{
P. Mardegan \\ (C) SFMU et Springer-Verlag France 2013
}

Comment, dans le contexte actuel, répondre à une grande majorité des français qui disent leur attachement à l'accessibilité et à la proximité [1] ? Comment garantir un accès à des soins de qualité en médecine d'urgence en moins de 30 minutes ? Un renforcement des moyens [2,3] sera nécessaire (ressources humaines, structures, techniques). Mais le principe de renforcement ne peut être à lui seul la solution. Il faudra aussi développer des coopérations entre les structures d'urgence et favoriser la mise en place d'équipes médicales de territoires [3].

L'hôpital a vécu, au cours de ces dix dernières années, des réformes du financement de son fonctionnement, particulièrement celle de la tarification à l'activité (T2A), qui a conduit nos établissements à piloter par la ressource. Les urgentistes de France ont su faire preuve d'initiatives innovantes, de recherche de la qualité pour la meilleure prise en charge, ont su accepter de se confronter à l'analyse de la performance dans un contexte de réformes successives des hôpitaux. Nous savons, aujourd'hui, que l'accès à une médecine d'urgence de qualité est étroitement lié au bon niveau des pratiques médicales des équipes, au contexte et aux particularités géographiques, et bien sûr, à un financement adapté à l'activité. L'hôpital reste sous contrainte (contrat pluriannuel d'objectifs et de moyens, T2A, l'état des prévisions des recettes et des dépenses, recherche, enseignement, performance...), et doit faire face à une augmentation constante de la consommation en soins urgents (appels au Samu, nombre de passages aux urgences). En période de crise, on est en droit de se demander si les urgences coûtent cher, voire « trop » cher [4] ? Ou, en définitive, pas assez cher, car leur financement n'est plus en rapport avec leur activité ?

Combien coûte vraiment le fonctionnement des structures d'urgence en France ? C'est-à-dire quels sont les financements prévus, chaque année, pour faire fonctionner 650 services d'urgence, 400 Smur et $98 \mathrm{Samu} .$. : la réponse est

P. Mardegan $(\bowtie)$

Pôle réanimation, anesthésie, urgences, Samu,

Centre hospitalier de Montauban, 100, boulevard Léon Cladel,

F-82000 Montauban, France

e-mail : p.mardegan@ch-montauban.fr
4 milliards d'euros.... Ces 4 milliards d'euros se décomposent de la façon suivante :

- les missions d'intérêt général (MIG) [5] :

- MIG Samu : 214 millions d'euros ;

- MIG Smur + recettes titre 2:617 MIG + 129 T2 $=746$ millions d'euros.

Soit un total de 1 milliard d'euros pour faire fonctionner les Samu et les Smur, permettant d'assurer à la quasi-totalité de la population sur l'ensemble du territoire le recours à un service d'urgence en moins de 30 minutes.

- Services d'urgence [5]:

- recettes directes par le forfait des urgences (FAU) : 950 millions d'euros ;

- forfait accueil et traitement des urgences (ATU) : 300 millions d'euros

- actes / consultations : 530 millions d'euros ;

- groupes homogènes de séjour (GHS) des unités d'hospitalisation de courte durée (UHCD) (mono-séjours) : 435 millions d'euros;

- ticket modérateur : 200 millions d'euros ;

- autres recettes (aide à la contractualisation...) : 70 millions d'euros.

Soit un total de 2,5 milliards d'euros pour les services d'urgence.

Ce montant reste incomplet si on ne rajoute pas à cela une estimation des recettes correspondantes au nombre de passages aux urgences suivis d'hospitalisation en services médecine-chirurgie-obstétrique (MCO) [6,7]. Ces recettes sont réputées être financées par les GHS/MCO et seraient de l'ordre de 550 millions. Il convient d'ailleurs de souligner que ces recettes ne sont pas toujours imputées aux services des urgences et à leur pôle correspondant, ce qui constitue une anomalie.

À première vue, on pourrait s'interroger sur le coût du fonctionnement des structures d'urgence. Or, ce montant ne représente qu'environ $2 \%$ des dépenses globales de santé en France [8] pour traiter 18 millions de passages aux urgences, 
pour répondre à 20 millions d'appels au Samu-centre 15 (avec 12 millions de dossiers de régulation) et pour réaliser près d'un million de missions Smur...[3]. Cette somme de 4 milliards d'euros représente un peu plus de 5 euros/mois/ habitant en France pour faire fonctionner nos structures d'urgence. La société paie deux fois plus pour les soins dentaires et dépense autant pour les transports sanitaires. Les coûts de gestion de la sécurité sociale et des complémentaires santé sont d'environ 14 milliards d'euros [8] !

L'heure du choix est devant nous : politique, économique, sanitaire ? Les trois probablement... Il semble, aujourd'hui, que les urgences ne coûtent pas si cher par rapport au service rendu. Non seulement une diminution de l'allocation budgétaire est inimaginable, mais un renforcement des moyens dans certaines structures sera probablement nécessaire. Les pouvoirs publics doivent pouvoir évaluer par exemple quel effectif est nécessaire pour prendre en charge tel volume d'activité dans des conditions optimales de sécurité et de qualité des soins et quel est le financement nécessaire correspondant. Les recommandations de Samu Urgences de France, dans le cadre du référentiel sur les ressources médicales et non médicales des Samu, Smur et services d'urgence, proposent une approche de ce dimensionnement en rapport avec l'activité [2]. Ce n'est qu'ensuite que l'on pourra alors dire si on donne trop ou pas assez pour les urgences!

Une réflexion sur l'évolution du financement implique une analyse « critique » du modèle actuel. Par exemple pour les Samu, à activité égale (ou missions équivalentes) on observe des « écarts » difficilement compréhensibles, voire difficilement acceptables sous prétexte de " dotations historiques $» . .$. même si une partie des écarts est explicable (politique régionale...). Soit on reste dans une approche strictement budgétaire, continuant à payer " en aveugle ", en espérant que les dépenses " n'explosent» pas, et que l'on reste dans " l'enveloppe " (c'est la crise, on gère au cas par cas, et on attend que ça passe ...). Soit il est temps d'objectiver vraiment ce que l'on finance, et de faire des choix (politique, économique et sanitaire) pour répondre autant aux besoins de la population en soins urgents qu'aux attentes des professionnels.

Ces questions attendent une réponse. On ne peut pas imaginer que la décision politique soit celle du « non choix ». Les médecins connaissent bien l'impact du « non choix », et ses conséquences prévisibles dans le domaine de la sécurité et de la qualité des soins, mais aussi le prix à payer des mauvais choix du passé ! Les urgentistes doivent être partie prenante dans ces réflexions sur les perspectives d'évolution, sur le financement comme sur l'organisation des structures d'urgence, afin d'éclairer le débat et de participer au choix de façon constructive.

On sait aujourd'hui que les urgences préhospitalières et hospitalières ne génèrent pas de surcoût pour la société, au contraire $[5,6,8]$. Il est temps de clarifier et de rendre lisible les règles de financement pour une allocation plus transparente et concertée des ressources (FAU, MIG...) afin d'assurer le fonctionnement des activités des structures d'urgence pour leur mission de service public.

\section{Références}

1. « Les français et l'hôpital » Sondage TNS-SOFRES du 27 au 30 mars 2013 pour la Fédération Hospitalière de France. Salon de la santé et de l'autonomie, 21 mai 2013, Paris

2. Samu-Urgences de France. Référentiel : les ressources médicales et non médicales nécessaires au bon fonctionnement des structures d'urgence (Samu, Smur, Service des Urgences, UHCD), novembre 2011. http://www.samu-urgences-de-France.fr (dernier accès 21 août 2013)

3. Mardegan P, Direction générale de l'offre de soins (DGOS) (2010) Rapport de la mission DGOS relative à la modernisation des SAMU. État des lieux, objectifs et recommandations opérationnelles, juillet 2010. http://www.dgos.sante.gouv.fr

4. Bohineust A (2013) Urgences : un coût de 1,5 millions par an. Le Figaro, vendredi 18 juillet 2013. http://Sante.lefigaro/actualite/ 2013/07/18/20999(dernier accès 12 août 2013)

5. Direction générale de l'offre de soins (DGOS). Financement des activités de médecine d'urgence. État des lieux et constats. Document transmis par le Ministère de la Santé. Groupe technique financement des structures d'urgence, 9 décembre 2011

6. Direction générale de l'offre de soins (DGOS). Financement des activités de médecine d'urgence. État des lieux et constats. Document transmis par le ministère de la santé. Groupe technique financement des structures d'urgence, 16 mars 2012

7. Mission nationale d'expertise et d'audit hospitaliers (MEAH). Nouvelle gouvernance et comptabilité analytique par pôle. Annexe 5 : méthodologie de calcul de la part des GHS à reverser aux urgences http://www.anap.fr/uploads/tx_sabasedocu/Nouvelle_ gouvernance_et_compta_analytique_2009.pdf (dernier accès le 21 août 2013)

8. Direction de la recherche, des études, de l'évaluation et des statistiques (DREES). Comptes nationaux de la santé 2011. http://www. drees.sante.gouv.fr/IMG/pdf/seriestat172.pdf (dernier accès le 21 août 2013) 